\title{
Search for s-channel single top-quark production in pp collisions
}

\author{
Mario Merola* for the CMS Collaboration \\ INFN, Napoli (IT) \\ E-mail: mario.merola@cern.ch
}

\begin{abstract}
A search for single top-quark production in the s channel in proton-proton collisions at a centre-ofmass energy of $\sqrt{s}=8 \mathrm{TeV}$ by the CMS detector at the LHC is presented. Leptonic decay modes of the top quark with an electron or muon in the final state are considered. The signal is extracted by performing a maximum-likelihood fit to the distribution of a multivariate discriminant defined using Boosted Decision Trees to separate the expected signal contribution from the background processes. Data collected in 2012, corresponding to an integrated luminosity of $19.3 \mathrm{fb}^{-1}$, leads to an upper limit on the cross section times branching ratio of $11.5 \mathrm{pb}$ at $95 \%$ confidence level.
\end{abstract}

The European Physical Society Conference on High Energy Physics 22-29 July 2015

Vienna, Austria

${ }^{*}$ Speaker. 


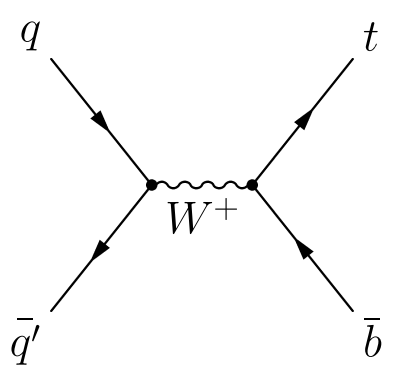

Figure 1: Leading order (LO) Feynman diagram for single top quark production in the $s$ channel.

\section{Introduction}

The top quark, discovered at the Tevatron proton anti-proton collider in 1995 [1, 2], can be produced via strong interaction in association with the top anti-quark and via electroweak interaction as single top. Three are the different single top production modes, the $t$ channel, the $s$ channel and the tW production. The single top production in the $s$ channel at the LHC is unfavoured with respect to the other two production modes, involving a quark and an anti-quark in the initial state (Figure 1). The SM prediction at NNLL (next-to-next-to-leading logarithmic) order for this process at the LHC and at $8 \mathrm{TeV}$ is $\sigma_{s-\text { th. }}^{\text {th }}=5.55 \pm 0.08$ (scale) \pm 0.21 (PDF) pb [6]. Nonetheless the interest in this channel is focused on possible enhancement of its production due to new physics involving non-SM mediators, like $\mathrm{W}^{\prime}$ or charged Higgs boson [8].

The study presented here [11] analyses $19.3 \mathrm{fb}^{-1}$ of data collected by the CMS detector [3] in 2012 at a centre-of-mass energy $\sqrt{s}=8 \mathrm{TeV}$.

\section{Event selection and multivariate analysis}

The final state topology of the single top production in the $s$ channel is characterised by the presence of one muon or electron and two b quarks, one coming from the top-quark decay and one recoiling against the top quark. The event selection considers exactly one isolated muon or one electron with $p_{\mathrm{T}}>26 \mathrm{GeV} / c,|\eta|<2.1$ and $p_{\mathrm{T}}>30 \mathrm{GeV} / c,|\eta|<2.5$, respectively. Exactly two jets, reconstructed by clustering particle flow candidates [4] using the anti- $k_{T}$ algorithm [7], are required to have $p_{\mathrm{T}}>40 \mathrm{GeV} / c$ for $|\eta|<4.5$ and events with additional jets with $p_{\mathrm{T}}>30 \mathrm{GeV} / c$ are rejected. The two selected jets are also required to be originated from $b$ quarks, for which the track counting algorithm with high purity requirement [5] is adopted, restricting their acceptance to $|\eta|<2.5$. These cuts allow a tight rejection of $\mathrm{W}+$ jets and multijet QCD backgrounds. Due to the ambiguity in the reconstruction of the top quark hypothesis from $b$ jet, lepton and $E_{\mathrm{T}}$, two top mass hypotheses are defined and the correct $\mathrm{b}$ jet-to-top assignment is taken based on the one which gives the closer invariant top mass to the reference value $172.5 \mathrm{GeV} / c^{2}$.

The events passing the above selection define the signal region (henceforth $2 j 2 t$ ), while a control region, enriched in $\mathrm{t} \overline{\mathrm{t}}$ events, is defined selecting 3 jets with two of them passing b-tagging requirements ( $3 j 2 t$ event category).

At the end the signal sample is still dominated by the backgrounds, mainly $\mathrm{t} \overline{\mathrm{t}}, \mathrm{W}+\mathrm{jets}$ and $t$ channel single top, and so a multivariate technique using Boosted Decision Trees (BDT) [10] 

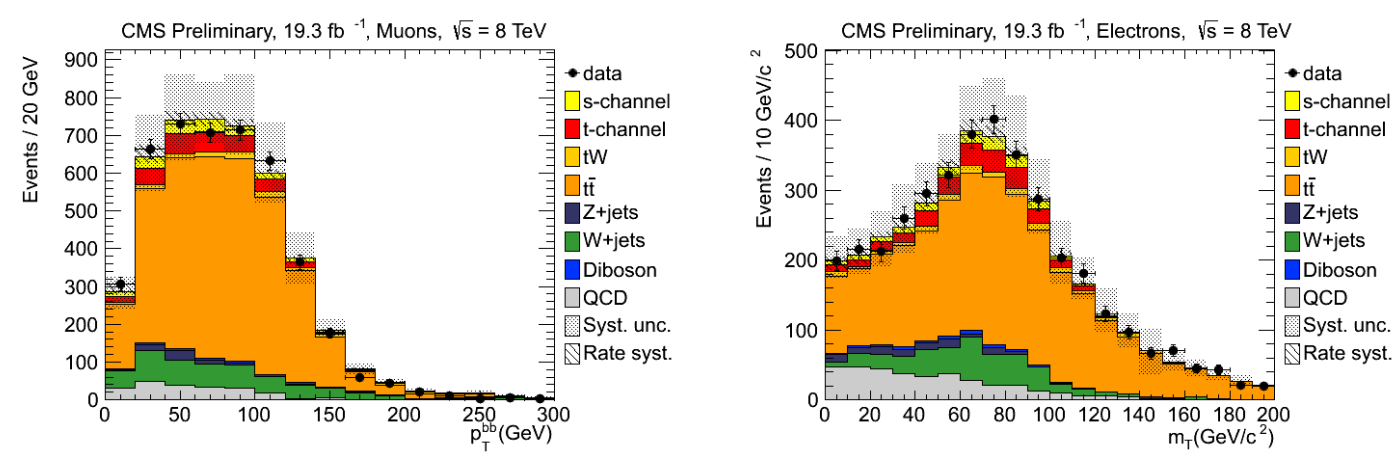

Figure 2: Two of the most discriminating variables used in the BDT to separate signal and backgrounds: vector sum of $p_{\mathrm{T}}$ of the two b-jets system (left) and the $\mathrm{W}$ boson transverse mass (right).

is performed in order to take advantage of the different final state topology of the signal and the backgrounds. Two independent BDT trainings are set up in the $2 j 2 t$ and $3 j 2 t$ event categories, optimised for electron and muon decay channels. Among the kinematic and angular variables considered, the ones with the highest discriminating power are the vector sum of $p_{\mathrm{T}}$ of the two b-jets system and the $\mathrm{W}$ boson transverse mass (Figure 2). For the first variable the signal shows a lower $p_{\mathrm{T}}$ distribution with respect to $\mathrm{t} \overline{\mathrm{t}}$ since the two $\mathrm{b}$ jets carry a larger fraction of the original transverse momentum which peaks around zero. The $\mathrm{W}$ transverse mass, reconstructed from the lepton and the $E_{T}$, shows a clear jacobian peak for lepton-neutrino pairs coming from the same W-boson decay (signal), while presents a broader distribution for events where two W bosons, both

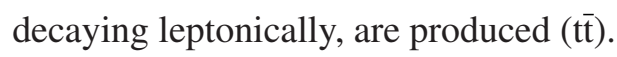

\section{Signal extraction and results}

The $s$-channel signal extraction consists in a binned maximum likelihood fit to the BDT distributions on data in the $2 j 2 t$ and $3 j 2 t$ categories simultaneously (Figure 3 ). The inclusion of the $3 j 2 t$ category allows to constrain the $t \bar{t}$ and $\mathrm{W}+$ jets backgrounds while taking into account all possible correlations in the systematics uncertainties between the two regions. The signal and all the background models are taken from simulation except for the multijet QCD background, for which a dedicated treatment has been adopted. The QCD model is taken from a control region in data defined inverting the lepton isolation for muons and requiring that either the isolation cut or identification criteria fail for electrons. The QCD normalisation is estimated by a fit to the $m_{\mathrm{T}}$ (muons) or $\mathscr{H}_{\mathrm{T}}$ (electrons) and its uncertainty is conservatively assigned by repeating the fit in a QCD-enriched region and varying the non-QCD contributions by $20 \%$.

The likelihood model includes the background rates as nuisance parameters with a coefficient for each background template with a log-normal prior. The prior uncertainties on the background normalisation have been taken from the recent measurements of the corresponding processes, i.e. $10 \%$ for $\mathrm{t} \overline{\mathrm{t}}, 30 \%$ for $\mathrm{W}+\mathrm{jets}, 10-30 \%$ for $\mathrm{QCD}$ (in the different event categories), $15 \%$ for single top in the $t$ channel. The impact of the background rates uncertainties on the $s$-channel cross section measurement is evaluated removing one uncertainty at a time from the likelihood model and measuring the corresponding variation in the profile likelihood. The other instrumental (as jet energy 

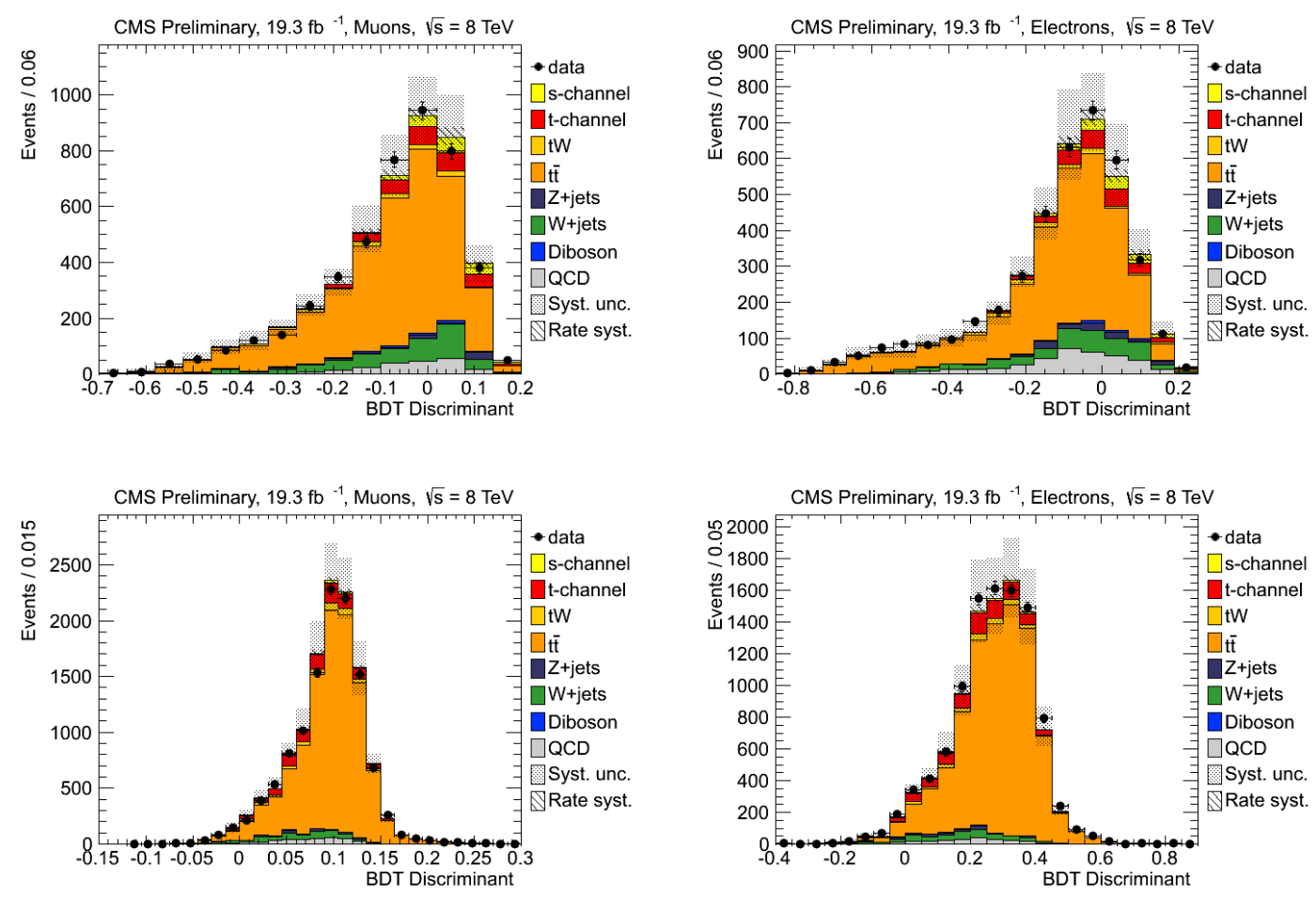

Figure 3: BDT discriminant for the muon (left) and electron (right) channels, in the signal (up) and $t \bar{t}$ enriched (down) regions. The simulation is normalised to the fit results.

scale and resolution, b-tagging efficiencies and pile-up) and theoretical (as renormalisation and factorisation scales, matrix element and parton showering matching thresholds, PDFs) uncertainties are evaluated performing two fits to the pseudodatasets generated with the up/down variations of the systematic source of interest (with other sources fixed to zero), and taking the difference in the fitted signal yield between these fits and the nominal fit as the corresponding up/down uncertainties.

The systematic uncertainties with the highest impact on the measurement have been found to be the factorisation and renormalisation scales $(\sim 80 \%)$, jet energy scales $(\sim 50 \%)$, and matching thresholds $(\sim 30 \%)$. The single top-quark $s$-channel inclusive production cross section has been measured to be $\sigma_{s-\text { ch. }}=6.2 \pm 5.4$ (exp.) \pm 5.9 (th.) pb. Using the Feldman-Cousins unified approach [9] the $68 \%$ confidence level (CL) intervals which do not reach negative cross section values are reported as well: $\sigma_{s-\mathrm{ch} .}=6.2_{-5.1}^{+8.0} \mathrm{pb}$. Finally, the measurement leads to an upper limit of 11.5 $(17.0,9.0) \mathrm{pb}$ at $95 \% \mathrm{CL}$, evaluated using a Bayesian approach, where the numbers in parenthesis refer to the expected limits in presence of SM signal and in the background-only hypothesis.

Future extensions of this analysis will include the whole $7+8 \mathrm{TeV}$ dataset to increase the sensitivity of the measurement, will expoit other background enriched phase space regions and the reduced factorisation/renormalisation scale uncertainties from the next-to-leading order $t \bar{t}$ generators. With the $13 \mathrm{TeV}$ centre-of-mass energy collisions the unfavourable signal over background ratio (the $\bar{t}$ cross section increases by a factor of 3 and the s channel by a factor of 2) will require a much larger dataset (about first two years of data taking) to be competitive with the measurement 
performed on Run 1 data.

\section{References}

[1] CDF Collaboration, Observation of Top Quark Production in $\bar{p} p$ Collisions with the Collider Detector at Fermilab, Phys. Rev. Lett. 74 (1995) 2626

[2] D0 Collaboration, Observation of the Top Quark, Phys. Rev. Lett. 74 (1995) 2632

[3] CMS Collaboration, The CMS experiment at the CERN LHC, JINST 03 (2008) S08004

[4] CMS Collaboration, Particle-Flow Event Reconstruction in CMS and Performance for Jets, Taus, and $E_{\mathrm{T}}$, CMS Physics Analysis Summary CMS-PAS-PFT-09-001 (2009)

[5] CMS Collaboration, b-Jet Identification in the CMS Experiment, CMS Physics Analysis Summary CMS-PAS-BTV-11-004 (2011)

[6] Kidonakis, N., NNLL threshold resummation for top-pair and single-top production, Phys. Part. Nucl. 45 (2014) 714 [hep-ph/1210 . 7813]

[7] Cacciari, M. and Salam, G. P. and Soyez, G., The anti-k t jet clustering algorithm, JHEP 04 (2008) 063

[8] Tait, T. M. P. and Yuan, C. P., Single top quark production as a window to physics beyond the standard model, Phys. Rev. D 63 (2000) 014018

[9] Feldman, G. J. and Cousins, R. D., Unified approach to the classical statistical analysis of small signals, Phys. Rev. D 57 (1998) 3873

[10] Breiman, L. and Friedman, J. and Stone, C. J. and Olshen, R. A., Classification and Regression Trees, Chapman and Hall, 1984.

[11] CMS Collaboration, Search for s-channel single top-quark production in pp collisions at $\sqrt{s}=8 \mathrm{TeV}$, CMS Physics Analysis Summary CMS-PAS-TOP-13-009 (2013) 